\title{
FACIAL INCIDENCE COLORINGS OF EMBEDDED MULTIGRAPHS $^{1}$
}

\author{
Mirko HorŇÁK, Stanislav Jendrol' \\ AND \\ ROMAN SOTÁK \\ Institute of Mathematics \\ P.J. Šafárik University \\ Jesenná 5, 04001 Košice, Slovakia \\ e-mail: \{mirko.hornak, stanislav.jendrol, roman.sotak\}@upjs.sk
}

\begin{abstract}
Let $G$ be a cellular embedding of a multigraph in a 2-manifold. Two distinct edges $e_{1}, e_{2} \in E(G)$ are facially adjacent if they are consecutive on a facial walk of a face $f \in F(G)$. An incidence of the multigraph $G$ is a pair $(v, e)$, where $v \in V(G), e \in E(G)$ and $v$ is incident with $e$ in $G$. Two distinct incidences $\left(v_{1}, e_{1}\right)$ and $\left(v_{2}, e_{2}\right)$ of $G$ are facially adjacent if either $e_{1}=e_{2}$ or $e_{1}, e_{2}$ are facially adjacent and either $v_{1}=v_{2}$ or $v_{1} \neq v_{2}$ and there is $i \in\{1,2\}$ such that $e_{i}$ is incident with both $v_{1}, v_{2}$. A facial incidence coloring of $G$ assigns a color to each incidence of $G$ in such a way that facially adjacent incidences get distinct colors. In this note we show that any embedded multigraph has a facial incidence coloring with seven colors. This bound is improved to six for several wide families of plane graphs and to four for plane triangulations.
\end{abstract}

Keywords: embedded multigraph, incidence, facial incidence coloring.

2010 Mathematics Subject Classification: 05C15, 05C10.

\section{REFERENCES}

[1] I. Algor and N. Alon, The star arboricity of graphs, Discrete Math. 75 (1989) 11-22. doi:10.1016/0012-365X(89)90073-3

\footnotetext{
${ }^{1}$ The research was supported by the Slovak grant VEGA $1 / 0368 / 16$, by the grant APVV15-0116, and by the Agency of the Ministry of Education, Science, Research and Sport of the Slovak Republic for the Structural Funds of EU under the project ITMS 26220120007.
} 
[2] K. Appel and W. Haken, Every Planar Map is Four Colorable (Contemporary Mathematics Vol. 98, American Mathematical Society, Providence, Rhode Island, 1989).

[3] M. Axenovich, T. Ueckerdt and P. Weiner, Splitting planar graphs of girth 6 into two linear forests with short paths, J. Graph Theory 85 (2017) 601-618. doi:10.1002/jgt.22093

[4] M. Bonamy, B. Lévêque and A. Pinlou, 2-distance coloring of sparse graphs, J. Graph Theory 77 (2014) 190-218. doi:10.1002/jgt.21782

[5] R.A. Brualdi and J.J. Quinn Massey, Incidence and strong edge colorings of graphs, Discrete Math. 122 (1993) 51-58. doi:10.1016/0012-365X(93)90286-3

[6] I. Fabrici, S. Jendrol' and M. Vrbjarová, Unique-maximum edge-colouring of plane graphs with respect to faces, Discrete Appl. Math. 185 (2015) 239-243. doi:10.1016/j.dam.2014.12.002

[7] W. Goddard, Acyclic colorings of planar graphs, Discrete Math. 91 (1991) 91-94. doi:10.1016/0012-365X(91)90166-Y

[8] B. Guiduli, On incidence coloring and star arboricity of graphs, Discrete Math. 163 (1997) 275-278. doi:10.1016/0012-365X(95)00342-T

[9] P. Hall, On representatives of subsets, J. Lond. Math. Soc. 10 (1935) 26-30. doi:10.1112/jlms/s1-10.37.26

[10] M. Hosseini Dolama, É. Sopena and X. Zhu, Incidence coloring of $k$-degenerated graphs, Discrete Math. 283 (2004) 121-128. doi:10.1016/j.disc.2004.01.015

[11] M. Hosseini Dolama and É. Sopena, On the maximum average degree and the incidence chromatic number of a graph, Discrete Math. Theor. Comput. Sci. 7 (2005) 203-216.

[12] X. Li and J. Tu, NP-completeness of 4-incidence colorability of semicubic graphs, Discrete Math. 308 (2008) 1334-1340. doi:10.1016/j.disc.2007.03.076

[13] M. Maydanskyi, The incidence coloring conjecture for graphs of maximum degree 3 , Discrete Math. 292 (2005) 131-141. doi:10.1016/j.disc.2005.02.003

[14] K.S. Poh, On the linear vertex-arboricity of a planar graph, J. Graph Theory 14 (1990) 73-75. doi:10.1002/jgt.3190140108

[15] N. Robertson, D. Sanders, P. Seymour and R. Thomas, The four-colour theorem, J. Combin. Theory Ser. B 70 (1997) 2-44. doi:10.1006/jctb.1997.1750 
[16] W.C. Shiu, P.C.B. Lam and D.L. Chen, On incidence coloring for some cubic graphs, Discrete Math. 252 (2002) 259-266. doi:10.1016/S0012-365X(01)00457-5

[17] W.C. Shiu and P.K. Sun, Invalid proofs on incidence coloring, Discrete Math. 308 (2008) 6575-6580. doi:10.1016/j.disc.2007.11.030

[18] P.K. Sun, Incidence coloring of regular graphs and complement graphs, Taiwanese J. Math. 16 (2012) 2289-2295. doi:10.11650/twjm/1500406852

[19] W.F. Wang and K.W. Lih, Labeling planar graphs with conditions on girth and distance two, SIAM J. Discrete Math. 17 (2003) 264-275. doi:10.1137/S0895480101390448

[20] J. Wu, Some results on the incidence coloring number of a graph, Discrete Math. 309 (2009) 3866-3870. doi:10.1016/j.disc.2008.10.027

[21] D. Yang, Fractional incidence coloring and star arboricity of graphs, Ars Combin. 105 (2012) 213-224.

Received 12 December 2016 Revised 17 May 2017 Accepted 17 May 2017 